\title{
Synthesis of Structurally Well-Defined and Liquid-Phase-Processable Graphene Nanoribbons
}

Akimitsu Narita ${ }^{1}$, Xinliang Feng ${ }^{1} *$, Yenny Hernandez ${ }^{1}$, Søren A. Jensen ${ }^{1,2}$, Mischa Bonn ${ }^{1}$, Huafeng Yang ${ }^{3}$, Ivan A. Verzhbitskiy ${ }^{4}$, Cinzia Casiraghi, ${ }^{3,4}$, Michael Ryan Hansen ${ }^{1,5}$, Amelie H. R. Koch ${ }^{1}$, George Fytas ${ }^{1,6}$, Oleksandr Ivasenko ${ }^{7}$, Bing Li ${ }^{7}$, Kunal S. Mali ${ }^{7}$, Tatyana Balandina ${ }^{7}$, Sankarapillai Mahesh ${ }^{7}$, Steven De Feyter ${ }^{7}$, Klaus Müllen ${ }^{1 *}$

${ }^{1}$ Max Planck Institute for Polymer Research, Ackermannweg 10, D-55128 Mainz, Germany.

${ }^{2}$ FOM Institute AMOLF, Science Park 104, 1098 XG Amsterdam, The Netherlands.

${ }^{3}$ School of Chemistry and Photon Science Institute, Manchester University, Oxford Road, Manchester, M139PL, United Kingdom.

${ }^{4}$ Department of Physics, Free University Berlin, Arnimalle 14, 14195 Berlin, Germany.

${ }^{5}$ Interdisciplinary Nanoscience Center (iNANO) and Department of Chemistry, Aarhus University, Gustav Wieds Vej 14, DK-8000 Aarhus C, Denmark.

${ }^{6}$ Department of Materials Science, University of Crete and FORTH, Heraklion, Greece.

${ }^{7}$ Division of Molecular Imaging and Photonics, Department of Chemistry, KU Leuven Celestijnenlaan, 200 F, B-3001 Leuven, Belgium.

*email: feng@mpip-mainz.mpg.de,muellen@mpip-mainz.mpg.de

\begin{abstract}
Graphene nanoribbons (GNRs) are excellent candidates for next-generation electronic materials. Unlike GNRs produced by "top-down" methods such as lithographical patterning of graphene and unzipping of carbon nanotubes that cannot reach structural perfection, the fabrication of structurally well-defined GNRs has been achieved by a "bottom-up" organic synthesis via solution-mediated or surface-assisted cyclodehydrogenation. Specifically, non-planar polyphenylene precursors were first "build up" from small molecules, and then "graphitized" and "planarized" to yield GNRs. However, fabrication of processable and longitudinally well-extended GNRs has remained a major challenge. Here we report a "bottom-up" solution synthesis of long (>200 nm), liquid-phase processable GNRs with well-defined structure and a large optical bandgap of $1.88 \mathrm{eV}$. Scanning probe microscopy demonstrates self-assembled monolayers of GNRs, and non-contact, time-resolved Terahertz conductivity measurements reveal excellent charge-carrier mobility within individual GNRs. Such structurally well-defined GNRs offer great opportunities for fundamental studies into graphene nanostructures, as well as development of GNR-based nanoelectronics.
\end{abstract}


Graphene nanoribbons (GNRs), defined as nanometre-wide strips of graphene, are attracting increasing attention as highly promising candidates for next generation semiconductor materials ${ }^{1,2,3,4}$. Quantum confinement effects impart GNRs with semiconducting properties, i.e. with a finite bandgap, which critically depends on the ribbon width and its edge structure ${ }^{1,3}$. Fabrication of GNRs has been primarily carried out by "top-down" approaches such as lithographical patterning of graphene $e^{5,6}$ and unzipping of carbon nanotubes ${ }^{7,8}$, revealing their semiconducting nature and excellent transport properties ${ }^{1}$. However, these methods are generally limited by low yields and lack of structural precision, leading to GNRs with uncontrolled edge structures.

In contrast, a "bottom-up" chemical synthetic approach based on solution-mediated ${ }^{9,10,11,12,13}$ or surface-assisted ${ }^{14}$ cyclodehydrogenation, namely "graphitization" and "planarization", of tailor-made three-dimensional polyphenylene precursors offers an appealing strategy for making structurally well-defined and homogeneous GNRs. The polyphenylene precursors are built up from small molecules, and thus their structures can be tailored within the capabilities of modern synthetic chemistry ${ }^{15}$. However, GNRs $(>30 \mathrm{~nm})$ produced by solution-mediated methods have been precluded from unambiguous structural characterization, i.e. microscopic visualization, due to their limited processability ${ }^{9,12}$. On the other hand, GNRs produced by the surface-assisted protocol have been characterized to be atomically precise using scanning tunnelling microscopy (STM) ${ }^{14}$. Nevertheless, this method can only provide a limited amount of GNR material, which is further bound to a metal surface, impeding wider applications in electronic devices.

There is hitherto no report on the large-scale fabrication of long (>100 nm) and processable GNRs with high structural definition. Here we demonstrate an efficient "bottom-up" solution synthesis of longitudinally well-extended (>200 nm) GNRs with excellent liquid-phase processability based on the cyclodehydrogenation of high-molecular-weight, semirigid polyphenylene precursors (Fig. 1a). The narrow GNRs had an optical bandgap of $1.88 \mathrm{eV}$, and their structure was supported by infrared, Raman, UV-vis absorption, and NMR spectroscopies. Scanning probe microscopy (SPM) analysis of GNRs deposited from dispersions on graphite substrates demonstrates an exquisitely ordered self-assembled monolayer of GNRs, further corroborating their well-defined structure. Moreover, such GNRs offer the chance for the first liquid-phase investigation of their electronic properties by employing non-contact, time-resolved Terahertz $(\mathrm{THz})$ conductivity measurements ${ }^{16}$, revealing excellent intramolecular charge-carrier mobility of the GNRs.

\section{Results and discussion}

Solution synthesis of liquid-phase processable GNRs with high longitudinal extension. First, a novel type of polyphenylene precursor 2 with extremely high molecular weight was synthesized by employing Diels-Alder polymerization (Fig. 1a) ${ }^{17,18}$. The monomer building block 1, consisting of a cyclopentadienone as the conjugated diene and an ethynyl group as the

DOI: 10.1038/NCHEM.1819 http://www.nature.com/nchem/journal/v6/n2/abs/nchem.1819.html 
dienophile, functions as a precursor for the $A B$-type Diels-Alder polymerization to yield $\mathbf{2}$ in high yield (Supplementary Methods). Dodecyl chains were installed on the periphery of $\mathbf{2}$ to enhance the dispersibility of the resulting GNRs as well as to sterically hinder undesired conformations, ensuring the formation of targeted GNRs upon the "graphitization" (Supplementary Fig. 1).

The polymerization of monomer 1 was performed simply by heating 1 to $260-270{ }^{\circ} \mathrm{C}$, without any additional reagent or catalyst, either in a diphenyl ether $\left(\mathrm{Ph}_{2} \mathrm{O}\right)$ solution or in a melt. Polyphenylene precursor 2 was most probably obtained as a mixture of regioisomers due to two possible orientations of unsymmetrical monomer 1 upon each Diels-Alder cycloaddition step. However, all the regioisomers led to one GNR structure as depicted in Supplementary Fig. 1. Based on size exclusion chromatography (SEC) analysis against polystyrene (PS) standards, the resulting weight-average molecular weight $\left(M_{\mathrm{w}}\right)$ of precursor 2 ranged from $(24 \pm 2)$ to $(620 \pm 60)$ $\mathrm{kgmol}^{-1}$, which was one order of magnitude larger than the $M_{\mathrm{w}}$ obtained by Ni-catalysed $A A$-type Yamamoto polymerization ${ }^{12}$, and surpassed the $M_{\mathrm{w}}$ of any reported linear polyphenylene polymers (Supplementary Table 1$)^{9,17,18} . M_{\mathrm{w}}$ higher than $100 \mathrm{kgmol}^{-1}$ was obtained when the concentration of monomer 1 was higher than $200 \mathrm{mM}$ in the polymerization. The polydispersity index (PDI) of precursor 2 ranged from 3.0 to 14 based on PS standard, which could be explained by the possible formation of cyclic oligomers in addition to polymers (Supplementary Figs. 2-5) ${ }^{19}$. Such small oligomers could be removed by fractionation with recycling preparative SEC or reprecipitation (Supplementary Fig. 5), often resulting in polymer precursors 2 with PDI of 1.9 or smaller (Supplementary Table 1).

Although the SEC analysis of such polyphenylene polymers with kinked and non-rigid backbone structures has been conventionally performed with PS standard ${ }^{9,12,13,17,18}$, it is reasonable to employ poly ( $p$-phenylene) (PPP) standard in parallel to obtain a second estimate of the actual molecular weight. For example, $M_{\mathrm{w}}$ of precursor 2-I was estimated based on PPP and PS standards to be $M_{\mathrm{w}, \mathrm{PPP}}$ : $(150 \pm 20) \mathrm{kgmol}^{-1}$ and $M_{\mathrm{w}, \mathrm{PS}}$ : $(380 \pm 40) \mathrm{kgmol}^{-1}$, respectively (Supplementary Table 1, entry 4'). Further, static light scattering analysis was performed for the unambiguous determination of absolute $M_{\mathrm{w}} \cdot{ }^{20}$ Dynamic light scattering experiment assured the presence of a single population of precursor 2 (Supplementary Fig. 6) and revealed its chain conformation (Fig. 1b) ${ }^{20}$. The absolute $M_{\mathrm{w}}$ of precursor 2-I was thus determined to be (470 \pm 30$)$ $\mathrm{kgmol}^{-1}$, which was well approximated by $M_{\mathrm{w}, \mathrm{PS}}$ (Fig. 1b). On the other hand, the absolute $M_{\mathrm{w}}$ of smaller-molecular-weight precursor 2-II $\left(M_{\mathrm{w}, \mathrm{PPP}}\right.$ : $(100 \pm 10) \mathrm{kgmol}^{-1}, M_{\mathrm{w}, \mathrm{PS}}$ : $(220 \pm 20) \mathrm{kgmol}^{-1}$, Supplementary Table 1 , entry 2') amounted to $108 \pm 8 \mathrm{kgmol}^{-1}$, which was closer to $M_{\mathrm{w}, \mathrm{PPP}}$. These results can be rationalized by the surprisingly large Kuhn length of precursor $2\left(l_{k} \sim 18 \mathrm{~nm}\right)$, i.e. precursor $\mathbf{2}$ assumes a semirigid conformation similar to PPP when it is short (2-II, $L_{\mathrm{w}}=$ ca. 110 $\mathrm{nm})$, but it behaves as semiflexible, being closer to PS, when it becomes longer (2-I, $L_{\mathrm{w}}=$ ca. $480 \mathrm{~nm}$ ) (vertical lines in Fig. 1b). It can therefore be concluded that the combination of PS and PPP standard calibration allows valid estimations of the $M_{\mathrm{w}}$ of precursor 2 . In the following text SEC data are given with ranges corresponding to those determined by PS and PPP standard calibration, e.g. $M_{\mathrm{w}, \mathrm{PPP}}-M_{\mathrm{w}, \mathrm{PS}}$. 
Finally, the non-planar precursor 2 was "planarized" and "graphitized" into GNR 3 in dichloromethane solution, employing intramolecular cyclodehydrogenation with iron (III) chloride as the Lewis acid and oxidant ${ }^{12,13}$. The synthesis can be readily scaled up to the gram scale. GNR 3 features a cove-type edge structure with a lateral width of $0.69-1.13 \mathrm{~nm}$ (Fig. 1a). Representatively, precursors 2-II with $M_{\mathrm{w}}$ of $(100 \pm 10)-(220 \pm 20) \mathrm{kgmol}^{-1}$ and PDI of 1.5-1.8 (Supplementary Table 1, entry 2') and 2-III with $M_{\mathrm{w}}$ of (270 \pm 30$)-(640 \pm 60) \mathrm{kgmol}^{-1}$ and PDI of 1.7-1.9 (Supplementary Table 1, entry 3') were transformed into GNRs 3-II and 3-III, respectively, which were identical within the realm of spectroscopic analysis (see below). The average length of GNRs 3-II and 3-III can be estimated to be ca. 90-200 and 250-600 nm, respectively, based on the $M_{\mathrm{w}}$ of precursors 2-II and 2-III.

For comparison, we also synthesized two model nanographene compounds, dimer $\mathbf{4}$ and trimer 5, which correspond to short cutouts of GNRs, from precursors $\mathbf{S 8}$ and S11, respectively (see Supplementary Methods and Supplementary Figs. 8-13). The successful "graphitization" of S8 and S11 into 4 and 5, respectively, was clearly demonstrated by matrix-assisted laser desorption/ionization time-of-flight (MALDI-TOF) mass spectrometry (MS) analysis, showing their isotopic distributions with no partially fused intermediates that were in perfect agreement with simulations (Supplementary Fig. 8). This result highlights the efficient "graphitization" of the oligophenylene precursors and thus supports the successful formation of GNR 3.

Spectroscopic characterization of the GNRs. The high efficiency of "graphitization" and "planarization" of precursor 2 into GNR 3 was confirmed by the combination of Fourier transform infrared (FTIR), Raman, and solid-state ${ }^{1} \mathrm{H}$ nuclear magnetic resonance (NMR) spectroscopies (Fig. 2 and Supplementary Figs. 7 and 15). FTIR analysis of precursor 2 and GNR 3-II, before and after the "graphitization", revealed the disappearance of out-of-plane (opla) C-H deformation bands at 698, 795, and $838 \mathrm{~cm}^{-1}$ which are typical for mono- and di-substituted benzene rings (Fig. $2 \mathrm{a}$ and Supplementary Fig. 15) ${ }^{12,21}$. Further, the signal triad from aromatic C-H stretching vibrations at 3025,3057 , and $3085 \mathrm{~cm}^{-1}$ were attenuated, and the opla band typical for aromatic C-H at the cove position (Fig. 1a) appeared at $863 \mathrm{~cm}^{-1}$, verifying the efficient conversion of precursor 2 into GNR $3^{9,12,21}$. These observations were in agreement with the changes observed in the FTIR spectra upon the cyclodehydrogenation of precursors $\mathbf{S 8}$ and S11 into dimer 4 and trimer 5, respectively (Supplementary Figs. 14 and 15).

The Raman spectrum of GNR 3-II (532 nm, powder sample) is consistent with those of GNRs previously synthesized by the bottom-up synthesis (Fig. 2b) ${ }^{12,14}$. The G peak is up-shifted $\left(\sim 1605 \mathrm{~cm}^{-1}\right)$ and has a larger Full Width at Half Maximum (FWHM 25 $\left.\mathrm{cm}^{-1}\right)$, which is expected from quantum confinement since this relaxes the Raman selection rule. The intense D peak is activated by the confinement of $\pi$-electrons into a finite-size domain, and described by collective modes of the confined hexagonal rings ${ }^{22}$, as shown by previous studies on polycyclic aromatic hydrocarbons (PAHs) ${ }^{23,24}$. Remarkably, a distinct peak from a ribbon width-specific low-frequency mode, namely the radial-breathing-like mode (RBLM), can be observed for GNR 3-II at $\sim 235 \mathrm{~cm}^{-1}$, which indicates high uniformity of the width of GNRs in the sample (Fig. 2b, inset) ${ }^{14}$. The low-frequency peak is visible with excitation at $532 \mathrm{~nm}$, but not at $633 \mathrm{~nm}$, showing its resonant nature similar to the radial-breathing mode (RBM) of CNTs. ${ }^{25}$ This peak is not DOI: 10.1038/NCHEM.1819 http://www.nature.com/nchem/journal/v6/n2/abs/nchem.1819.html 
observed in Raman spectra of dimer 4 and trimer 5, confirming that this peak is characteristic to the GNR structure (Supplementary Fig. 16). The wavenumber of the RBLM ( $v_{\text {RBLM }}$ ) is nearly independent of the edge structure and can be roughly estimated by $v_{\mathrm{RBLM}}=3222 / \mathrm{w}\left(\mathrm{cm}^{-1}\right)$, where $w(\AA)$ is the width of GNR ${ }^{26}$. The RBLM of GNR 3 can thus be estimated with the width of $w=$ $11.3 \AA$ to be $285 \mathrm{~cm}^{-1}$, which is in good agreement with the experimental result and further validates the formation of GNR 3 with uniform lateral width of $\sim 1 \mathrm{~nm}$.

The solid-state ${ }^{1} \mathrm{H}$ NMR characterization showed that precursor 2, which possesses a semiflexible to semirigid structure, becomes rigid after the "graphitization" to GNR $\mathbf{3}$ (Supplementary Fig. 7). Moreover, the $2 \mathrm{D}{ }^{1} \mathrm{H}-{ }^{1} \mathrm{H}$ double quantum-single quantum (DQ-SQ) NMR correlation experiments allowed the observation and assignment of the different protons at the cove positions for GNR 3. The significantly broadened ${ }^{1} \mathrm{H}$ signals for bulk GNR 3 can be ascribed to heterogeneous packing of GNRs, shifting the ${ }^{1} \mathrm{H}$ signals in opposite directions as a result of aromatic/anti-aromatic ring currents. ${ }^{27}$

Owing to the unique architecture of such GNRs with "Cove" edge periphery (Fig. 1a) and the dodecyl chains densely installed on the peripheral positions to alleviate the aggregation, GNR 3 displays excellent dispersibility in common organic solvents such as tetrahydrofuran (THF), chlorobenzene, and 1,2,4-trichlorobenzene (TCB). This dispersibility contrasts starkly with that of carbon nanotubes, graphene, and previously reported GNRs $(>30 \mathrm{~nm})$, which necessitate special highly polar solvent such as $N$-methylpyrrolidone (NMP) for exfoliation ${ }^{12,28}$. Mild sonication of GNR 3 in the aforementioned solvents generates transparent purple dispersions with a typical concentration of $\sim 0.01 \mathrm{mg} / \mathrm{mL}$ (Fig. 2c, inset). The dispersions are stable without any visible precipitation for at least three months. Albeit the presence of aggregates in dispersions, such remarkable dispersibility of GNR 3 in conventional organic solvents renders it liquid-phase processable and enables further physical characterization.

The UV-vis spectrum of GNR 3-II in an NMP dispersion reveals an absorption maximum at $550 \mathrm{~nm}$ and an optical bandgap of $1.88 \mathrm{eV}$ (Fig. 2c). GNRs 3-II and 3-III display basically identical absorption spectra. Clearly, for the 200-nm-long GNRs (3-I), the longitudinal confinement is negligible in determining the optical properties. UV-vis absorption spectra of dimer 4 and trimer 5 show similar spectral features to that of GNR 3-II with absorption maxima at 420 and $467 \mathrm{~nm}$, and optical bandgaps of 2.24 and $2.09 \mathrm{eV}$, respectively (Fig. 2c). These results indicate that the bandgap decreases with longitudinal extension to reach a plateau at 1.88 $\mathrm{eV}$. Moreover, the optical bandgap of $1.88 \mathrm{eV}$ is consistent with the estimated bandgap of 2.04 $\mathrm{eV}$, which was calculated by density functional theory ${ }^{29}$, further proving the structural identity of GNR 3.

SPM analyses of the GNRs. Profiting from the high dispersibility of GNR 3, we were able to image the molecular structure of such high molecular weight GNRs at the solid-air interface by means of STM. Figure 3a displays STM image of a dry film of GNR 3-II on highly oriented pyrolytic graphite (HOPG) deposited by leaching a small crystal using hot TCB. The STM image reveals straight and uniform GNRs co-aligned into domains with characteristic noodle-like structure similar to self-assemblies of conventional conjugated polymers such as polythiophenes

DOI: 10.1038/NCHEM.1819 http://www.nature.com/nchem/journal/v6/n2/abs/nchem.1819.html 
and polyanilines. The height information $(0.3 \sim 0.4 \mathrm{~nm})$ is indicative of the formation of self-assembled monolayers (Fig. $3 b)$. The average width of lamellae $(1.8 \pm 0.2 \mathrm{~nm})$ corresponds to only half of the calculated width of a single nanoribbon including the alkyl chains $(\sim 3.80 \mathrm{~nm})$, and suggests partial stacking interactions between neighbouring GNRs (Fig. 3b and 3c).

GNRs of up to ca. $60 \mathrm{~nm}$ can be readily imaged using such crystal leaching method. The prevalence of relatively shorter fragments of GNRs on the surface is due to the expected difference in the dispersibility of the shorter and longer GNRs. The relatively short GNRs are more dispersible than the longer ones and thus are preferentially extracted out during the deposition process. The longer fragments of GNRs within the sample are expected to be less dispersible for the obvious reasons, i.e. higher molecular weight and better stacking in the solid state, so they remain either in the crystals or as higher order (3D) aggregates. Indeed, GNR 3-III with larger average length showed no surface self-assembly at all, indicating necessity of higher dispersibility for the fabrication of self-assembled films consisting of longer ( $>100 \mathrm{~nm})$ GNRs. On the other hand, isolated ribbons could be visualized by STM at the Au(111)/TCB interface for short GNRs, i.e. ca. 14-27 nm (Supplementary Figs. 26 and 27). Remarkably enough, the periodicity observed in the edge structure of the GNRs was in full agreement with the longitudinal length of one repeating unit, providing further structural proof for GNR 3 (Supplementary Fig. 27).

To further enhance the dispersibility of GNRs, longer and branched 2-decyltetradecyl chains were installed on the peripheral positions of the GNRs (GNR 6, Fig. 3e). These alkyl chains are sterically bulkier than the dodecyl chains and have proven to be more effective at preventing the intermolecular $\pi-\pi$ stacking interactions of nanographenes ${ }^{30}$. The synthesis and characterization of GNR 6 was carried out in a similar manner to that of GNR 3, using

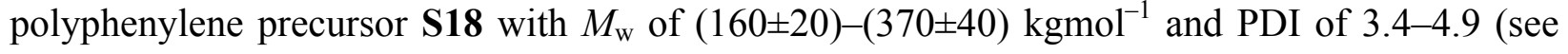
Supplementary Methods and Supplementary Figs. 17-24). The concentration of dispersions of GNR 6 in THF can reach $0.2 \mathrm{mg} / \mathrm{mL}$, which is over an order of magnitude higher than that of GNR 3. Moreover, GNR 6 is readily dispersible also in other conventional organic solvents such as chloroform and dichloromethane, in which GNR 3 shows no dispersibility.

Atomic force microscopy (AFM) visualization of a drop-casted film of GNR 6 on HOPG reveals highly ordered self-assembled monolayers of uniform GNRs with longitudinal length of over $200 \mathrm{~nm}$ (Fig. 3d). The observed length of the GNRs is in agreement with an estimated average length of ca. 110-260 nm calculated from the $M_{\mathrm{w}}$ of precursor S18. The measured width of the stripes is $4.1 \pm 0.1 \mathrm{~nm}$, corresponding to the calculated width of GNR 6 including the alkyl chains $(4.15 \mathrm{~nm}$ ) (Fig. $3 \mathrm{f}$ and $3 \mathrm{~g}$ ). The bulkiness and high density of branched alkyl chains substituted on these GNRs prevent the alkyl chain interdigitation and lead to formation of monolayers in which the molecules are simply adsorbed with their long axes parallel to each other. This packing arrangement is in contrast to that observed for GNR 3-II, where the monolayers were dominated by $\pi$-stacking interactions within coadsorbed molecules (Fig. $3 g$ ). The increased dispersibility of GNR 6 allowed the visualization of polymers of different lengths. AFM data obtained on the same sample often shows presence of longer and shorter GNRs deposited on the HOPG surface (Supplementary Fig. 25). These results evidently demonstrate

DOI: 10.1038/NCHEM.1819 http://www.nature.com/nchem/journal/v6/n2/abs/nchem.1819.html 
straight and long structure of GNRs 6 with excellent liquid-phase processability despite the presence of aggregates in dispersions.

Investigation of the electronic properties of GNRs by THz spectroscopy. The high dispersibility of GNRs enabled the unprecedented liquid-phase studies of their charge carrier mobility by utilizing time-resolved $\mathrm{THz}$ spectroscopy. While device fabrication on such narrow GNRs is highly challenging, this method allows simple and contact-free investigation of carrier mobility. To this end, we mobilized charges optically by above-bandgap excitation, using a short femtosecond pulse of $400 \mathrm{~nm}$ light. The conductivity of the charges was subsequently determined using a single cycle $\mathrm{THz}$ pulse. The $\mathrm{THz}$ pulses essentially constitute a very rapid succession of positive and negative fields (single-cycle pulses), which accelerate the charges. The interaction between the charges and the $\mathrm{THz}$ field modifies the transmitted $\mathrm{THz}$ pulse, which can be related directly to the complex (i.e. containing real and imaginary parts) conductivity of electrons and holes in the GNRs ${ }^{16}$. In contrast to device measurements that require charges to move over macroscopic distances, charges in our $\mathrm{THz}$ experiments are probed on ps timescales, during which they move only on the order of $10 \mathrm{~nm}$. Thus the intrinsic conduction properties of electrons in individual nanoribbons can be probed. The effect of the aggregation in the dispersion can be largely excluded as proved by $\mathrm{THz}$ spectroscopy investigation on drop-cast films of GNR 3 (Supplementary Fig. 29) and dispersions of dimer 4 (Supplementary Fig. 30).

Figure $4 \mathrm{a}$ shows the time-dependent conductivity of GNR 3-III in a TCB dispersion, which exhibits a fast rise after excitation and a subsequent rapid decay in both the real and the imaginary parts. The frequency-resolved complex photo-conductivity $\sigma(\omega ; \tau)$, recorded at $\tau=$ $300 \mathrm{fs}$ after photoinjection of charge carriers is shown in Fig. 4b. The conductivity shows significant positive real and negative imaginary components, which both increase in absolute value with frequency. Similar results were obtained for GNRs with shorter average length of ca. 20-33 nm (data not shown), indicating that the longitudinal confinement has a negligible influence on the $\mathrm{THz}$ conductivity.

It is instructive to compare THz-resolved conductivities of the GNR to those observed for organic semiconducting polymers such as polythiophene $(\mathrm{P} 3 \mathrm{HT})^{31,32}$ and poly-phenylenevinylene $(\mathrm{PPV})^{33,34}$ derivatives. There are clear similarities and striking differences. The conductivity spectrum $\sigma(\omega ; \tau)$ reported for the polymers is very similar to that shown in Fig. 4b. For the polymers, it was concluded by us $^{33,34}$ and others ${ }^{31,32}$ that such $\mathrm{THz}$ response is indicative of dispersive transport of free, unbound carriers with impeded long-range transport. These initially excited free carriers can quickly form excitons (free carrier lifetimes are on the order of 1 ps for the PPV derivatives ${ }^{33,34}$ ) as a result of the limited screening between electrons and holes in these low-dielectric systems.

Excitons are bound electrons and holes, which exhibit no real conductivity, but are highly polarizable. This polarizability appears in the conductivity spectrum as a negative imaginary conductivity that persists for long delay times, as also apparent for the GNRs (Fig. 4a) ${ }^{33}$. The time dependent conductivity observed in the GNRs (Fig. 4a) reveals an increase in the relative DOI: 10.1038/NCHEM.1819 http://www.nature.com/nchem/journal/v6/n2/abs/nchem.1819.html 
magnitude of the imaginary conductivity with time, consistent with the formation of excitons. The polarizability $\alpha$ can be determined from the slope of the imaginary conductivity response ${ }^{35}$ as it is expected that roughly every photon generates an exciton (quantum efficiency for exciton formation near $100 \%$ ) at long times ${ }^{34}$. The exciton polarizability amounts to $1.5 \times 10^{3} \AA^{3}$. This number is comparable to exciton polarizabilities previously determined for semiconducting polymers $^{34}$. These measurements thus show that to drive photocurrent in e.g. a photovoltaic device containing GNRs, exciton dissociation is required, analogous to semiconducting polymers, which are typically blended with fullerene derivatives to that purpose.

There are two notable features in the response of GNR 3-III that are distinct from those of conventional semiconducting polymers, namely (i) the relatively weak frequency dependence of the real conductivity and (ii) the large amplitude of the real conductivity. The frequency dependence of the real conductivity in polymers results from carriers interacting with torsional and conjugation irregularities along the polymer backbone. At higher frequencies the conductivity is probed over shorter length scales, where fewer irregularities are encountered, and thus higher conductivity is observed. Therefore, the weak frequency dependence observed for GNRs indicates a very low defect density and a mostly planar molecular geometry. Further, the amplitude of the real conductivity of GNRs, scaled to the excitation density, is almost an order of magnitude larger than what has been observed for semiconducting polymers in Ref. 34 .

The quantum efficiency for free carrier generation in polymer films and polymers in solution has been reported to be on the order of $10^{-3}$ and $10^{-5}$, respectively ${ }^{34}$. Accounting for this, we can estimate the carrier mobility of GNR 3-III measured in a dispersion to be within the range from 150 to $15,000 \mathrm{~cm}^{2} \mathrm{~V}^{-1} \mathrm{~s}^{-1}$ (See Supplementary Methods for details). Independent of the precise value of the mobility, it is clear that GNR possess conductivity properties superior to conventional organic polymeric semiconductors. The extracted mobility values are significantly lower than those of graphene reported to be as high as $200,000 \mathrm{~cm}^{2} \mathrm{~V}^{-1} \mathrm{~s}^{-1}$. ${ }^{36}$ This is to be expected when going to narrow ribbons since lateral confinement not only induces a bandgap, but also a nonzero effective carrier mass which scales linearly with the bandgap, dramatically reducing the carrier mobility ${ }^{37,38}$.

\section{Conclusion}

The strong aggregation nature of the GNRs hindered their comprehensive characterizations and rendered the interpretation of the obtained data difficult. Nevertheless, the high liquid-phase processability and the excellent intrinsic mobility indicated the remarkable potential of such bottom-up synthesized GNRs for future applications in nanoelectronics. The novel "bottom-up" solution-synthesis approach via Diels-Alder polymerization presented here is applicable to a variety of $A B$-type monomers, and thus holds a great promise for the tailor-made synthesis of long ( $>200 \mathrm{~nm}$ ), liquid-phase processable, and chemically precise GNRs with various width and edge structures. Moreover, installation of different functional groups is feasible on the periphery of such GNRs, which can bestow specific functionalities on them as well as further enhance their processability to realize wider applications such as in GNR-based nanocomposites. 


\section{Methods}

Sample preparation. Full details regarding the synthesis and characterization of all the materials are given in the Supplementary Information.

Raman spectroscopy analysis. Raman spectra were measured with a Witec Confocal Raman spectrometer, equipped with 633 and $532 \mathrm{~nm}$ laser lines. A 100X objective was used, and the power was kept well below $0.1 \mathrm{~mW}$ to avoid damage.

SPM visualization of GNRs. STM experiments were performed using PicoLE (Agilent) operating in constant-current mode on highly oriented pyrolytic graphite (HOPG, grade ZYB, Advanced Ceramics Inc.). STM tips were prepared by mechanical cutting from Pt/Ir wire $(80 \% / 20 \%$, diameter $0.2 \mathrm{~mm})$. A molecular model was constructed using HyperChem 7.0 program.* To form films of GNR 3, a small amount of the material was placed into a semi-closed capillary and fixed orthogonally over a preheated $\left(\sim 130{ }^{\circ} \mathrm{C}\right)$ HOPG surface. Application of small drops of TCB resulted in gradual etching of the GNR sample accompanied by solvent evaporation and radial deposition of dissolved nanoribbons onto a graphite surface in close vicinity to the crystal. For AFM measurements, a Multimode AFM with a Nanoscope IV controller (Veeco/Digital Instruments, Santa Barbara, USA) was employed in intermittent contact mode. Prior to AFM measurements, GNR powder was dispersed in TCB followed by heat and sonication cycles to ensure dissolution of GNRs. The AFM samples were then prepared by applying a drop of hot TCB dispersion on HOPG followed by evaporation of TCB at higher temperatures $\left(\sim 100^{\circ} \mathrm{C}\right)$. AFM as well as STM images were recorded at room temperature and were processed using SPIP ${ }^{\mathrm{TM}}$ (Image Metrology A/S, Denmark) software. STM images were calibrated using the graphite lattice as reference for obtaining correct lateral dimensions.

* HyperChem(TM) Professional 7.5, Hypercube, Inc., 1115 NW 4th Street, Gainesville, Florida 32601, USA

THz spectroscopy analysis of GNRs. Optical pump-THz probe measurements were performed using the output from a titanium sapphire laser generating pulses with a central wavelength of $800 \mathrm{~nm}$, and a duration of $\sim 40 \mathrm{fs}$, with a $1 \mathrm{kHz}$ repetition rate. Pump pulses were frequency doubled to $400 \mathrm{~nm}$ in a BBO (Barium Borate) crystal, and impinged on the sample after a mechanically adjustable delay. Single-cycle probe pulses comprising of frequencies in the $0-2$ THz range were generated from the $800 \mathrm{~nm}$ pulses in a $0.5 \mathrm{~mm}$ thick ZnTe crystal by a process called optical rectification ${ }^{16}$. After the sample, the transmitted $\mathrm{THz}$ field was detected with a third $800 \mathrm{~nm}$ pulse in a second ZnTe crystal using the electro-optic effect ${ }^{16}$. By mechanically delaying the detection pulse, the whole waveform of the $\mathrm{THz}$ pulse could be measured, and the frequency resolved conductivity could be obtained. 


\section{References and Notes:}

1. Li, X., Wang, X., Zhang, L., Lee, S. \& Dai, H. Chemically derived, ultrasmooth graphene nanoribbon semiconductors. Science 319, 1229-1232 (2008).

2. Castro Neto, A. H., Guinea, F., Peres, N. M. R., Novoselov, K. S. \& Geim, A. K. The electronic properties of graphene. Rev. Mod. Phys. 81, 109-162 (2009).

3. Ritter, K. A. \& Lyding, J. W. The influence of edge structure on the electronic properties of graphene quantum dots and nanoribbons. Nature Mater. 8, 235-242 (2009).

4. Son, Y. W., Cohen, M. L. \& Louie, S. G. Half-metallic graphene nanoribbons. Nature 444, 347-349 (2006).

5. Chen, Z., Lin, Y., Rooks, M. \& Avouris, P. Graphene nano-ribbon electronics. Physica E 40, 228-232 (2007).

6. Han, M., Özyilmaz, B., Zhang, Y. \& Kim, P. Energy Band-Gap Engineering of Graphene Nanoribbons. Phys. Rev. Lett. 98, 206805 (2007).

7. Kosynkin, D. V. et al. Longitudinal unzipping of carbon nanotubes to form graphene nanoribbons. Nature 458, 872-876 (2009).

8. Jiao, L., Zhang, L., Wang, X., Diankov, G. \& Dai, H. Narrow graphene nanoribbons from carbon nanotubes. Nature 458, 877-880 (2009).

9. Wu, J. S. et al. From branched polyphenylenes to graphite ribbons. Macromolecules 36, $7082-7089$ (2003).

10. Yang, X. Y. et al. Two-dimensional graphene nanoribbons. J. Am. Chem. Soc. 130, 4216-4217 (2008).

11. Fogel, Y. et al. Graphitic Nanoribbons with Dibenzo[e,1]pyrene Repeat Units: Synthesis and Self-Assembly. Macromolecules 42, 6878-6884 (2009).

12. Schwab, M. G. et al. Structurally Defined Graphene Nanoribbons with High Lateral Extension. J. Am. Chem. Soc. 134, 18169-18172 (2012).

13. Dössel, L., Gherghel, L., Feng, X. \& Müllen, K. Graphene nanoribbons by chemists: nanometer-sized, soluble, and defect-free. Angew. Chem. Int. Ed. 50, 2540-2543 (2011).

14. Cai, J. et al. Atomically precise bottom-up fabrication of graphene nanoribbons. Nature 466, 470-473 (2010).

15. Chen, L., Hernandez, Y., Feng, X. \& Müllen, K. From nanographene and graphene nanoribbons to graphene sheets: chemical synthesis. Angew. Chem. Int. Ed. 51, 7640-7654 (2012).

16. Ulbricht, R., Hendry, E., Shan, J., Heinz, T. \& Bonn, M. Carrier dynamics in semiconductors studied with time-resolved terahertz spectroscopy. Rev. Mod. Phys. 83, 543-586 (2011).

17. Shifrina, Z. B., Averina, M. S., Rusanov, A. L., Wagner, M. \& Müllen, K. Branched Polyphenylenes by Repetitive Diels-Alder Cycloaddition. Macromolecules 33, 3525-3529 (2000).

18. Kumar, U. \& Neenan, T. X. Diels-Alder Polymerization between Bis(cyclopentadienones) and Acetylenes. A Versatile Route to New Highly Aromatic Polymers. Macromolecules 28, 124-130 (1995). 
19. Kricheldorf, H. R. \& Schwarz, G. Cyclic Polymers by Kinetically Controlled Step-Growth Polymerization. Macromol. Rapid Commun. 24, 359-381 (2003).

20. Kroeger, A. et al. Equilibrium Length and Shape of Rodlike Polyelectrolyte Micelles in Dilute Aqueous Solutions. Macromolecules 40, 105-115 (2007).

21. Centrone, A. et al. Structure of new carbonaceous materials: The role of vibrational spectroscopy. Carbon 43, 1593-1609 (2005).

22. Castiglioni, C., Tommasini, M. \& Zerbi, G. Raman spectroscopy of polyconjugated molecules and materials: confinement effect in one and two dimensions. Philos. Transact. A Math. Phys. Eng. Sci. 362, 2425-2459 (2004).

23. Negri, F., Castiglioni, C., Tommasini, M. \& Zerbi, G. A Computational Study of the Raman Spectra of Large Polycyclic Aromatic Hydrocarbons: Toward Molecularly Defined Subunits of Graphite. J. Phys. Chem. A 106, 3306-3317 (2002).

24. Castiglioni, C., Mapelli, C., Negri, F. \& Zerbi, G. Origin of the D line in the Raman spectrum of graphite: A study based on Raman frequencies and intensities of polycyclic aromatic hydrocarbon molecules. J. Chem. Phys. 114, 963-974 (2001).

25. Saito, R., Hofmann, M., Dresselhaus, G., Jorio, A. \& Dresselhaus, M. S. Raman spectroscopy of graphene and carbon nanotubes. Adv. Phys. 60, 413-550 (2011).

26. Gillen, R., Mohr, M. \& Maultzsch, J. Symmetry properties of vibrational modes in graphene nanoribbons. Phys. Rev. B 81, 205426 (2010).

27. Chen, Z., Wannere, C. S., Corminboeuf, C., Puchta, R. \& von Ragué Schleyer, P. Nucleus-Independent Chemical Shifts (NICS) as an Aromaticity Criterion. Chem. Rev. 105, 3842-3888 (2005).

28. Hernandez, Y. et al. High-yield production of graphene by liquid-phase exfoliation of graphite. Nature Nanotechnol. 3, 563-568 (2008).

29. Beljonne, D. et al. Graphene Nanoribbons as Low-Band-Gap Donor Materials for Organic Photovoltaics: Quantum-Chemical Aided Design. ACS Nano, 6, 5539-5548 (2012).

30. Kastler, M., Pisula, W., Wasserfallen, D., Pakula, T. \& Müllen, K. Influence of alkyl substituents on the solution- and surface-organization of hexa-peri-hexabenzocoronenes. J. Am. Chem. Soc. 127, 4286-4296 (2005).

31. Ai, X. et al. Photoinduced charge carrier generation in a poly(3-hexylthiophene) and methanofullerene bulk heterojunction investigated by time-resolved Terahertz spectroscopy. J. Phys. Chem. B 110, 25462-25471 (2006).

32. Cunningham, P. D. \& Hayden, L. M. Carrier dynamics resulting from above and below gap excitation of P3HT and P3HT/PCBM investigated by optical-pump Terahertz-probe spectroscopy. J. Phys. Chem. C 112, 7928-7935 (2008).

33. Hendry, E., Schins, J. M., Candeias, L. P., Siebbeles, L. D. A. \& Bonn, M. Efficiency of Exciton and Charge Carrier Photogeneration in a Semiconducting Polymer. Phys. Rev. Lett. 92, 196601 (2004).

34. Hendry, E. et al. Interchain effects in the ultrafast photophysics of a semiconducting polymer: THz time-domain spectroscopy of thin films and isolated chains in solution. Phys. Rev. B 71, 125201 (2005). 
35. Wang, F. et al. Exciton polarizability in semiconductor nanocrystals. Nature Mater. 5, 861-864 (2006).

36. Bolotin, K. I. et al. Ultrahigh electron mobility in suspended graphene. Solid State Commun. 146, 351-355 (2008).

37. Obradovic, B. et al. Analysis of graphene nanoribbons as a channel material for field-effect transistors. Appl. Phys. Lett. 88, 142102 (2006).

38. Wang, J., Zhao, R., Yang, M., Liu, Z. \& Liu, Z. Inverse relationship between carrier mobility and bandgap in graphene. J. Chem. Phys. 138, 084701 (2013).

Acknowledgments This work was financially supported by ERC grant on NANOGRAPH, DFG Priority Program SPP 1355, DFG MU 334/32-1, DFG Priority Program SPP 1459, EU Project SUPERIOR (PITN-GA-2009-238177), UPGRADE, and GENIUS, BELSPO IAP-PAI network "Functional Supramolecular Systems" and Fund of Scientific Research - Flanders (FWO). We thank Louis Bertschi and Rolf Hafliger (LOC, ETH Zürich) for high-resolution MALDI-TOF MS analysis.

Author Contributions K. M. and X. F. planned the project. A.N. designed and synthesized all the materials and performed standard characterization, including FTIR analysis. A.N. and Y.H. conducted UV-vis absorption spectroscopic analysis. H.Y., I.A.V., and C.C. carried out Raman spectroscopic analysis. O.I., B.L., K.S.M., T.B., and S.M. performed SPM experiments. S.A.J. conducted the THz spectroscopy experiments. M.R.H carried out solid-state NMR experiments. A.H.R.K. performed laser light scattering experiments. X.F., M.B., G.F., S.D.F, and K.M. supervised the experiments. A.N., S.A.J., C.C., G.F., O.I., and K.S.M. co-wrote the manuscript, and X.F., M.B., S.D.F., and K.M. did the corrections and finalization. All authors discussed the results and implications and commented on the manuscript.

\section{Additional Information}

Supplementary information is available in the online version of the paper. Reprints and permissions information is available at www.nature.com/reprints. Correspondence and requests for materials should be addressed to X.F. or K.M.

\section{Competing financial interests}

The authors declare no competing financial interests. 

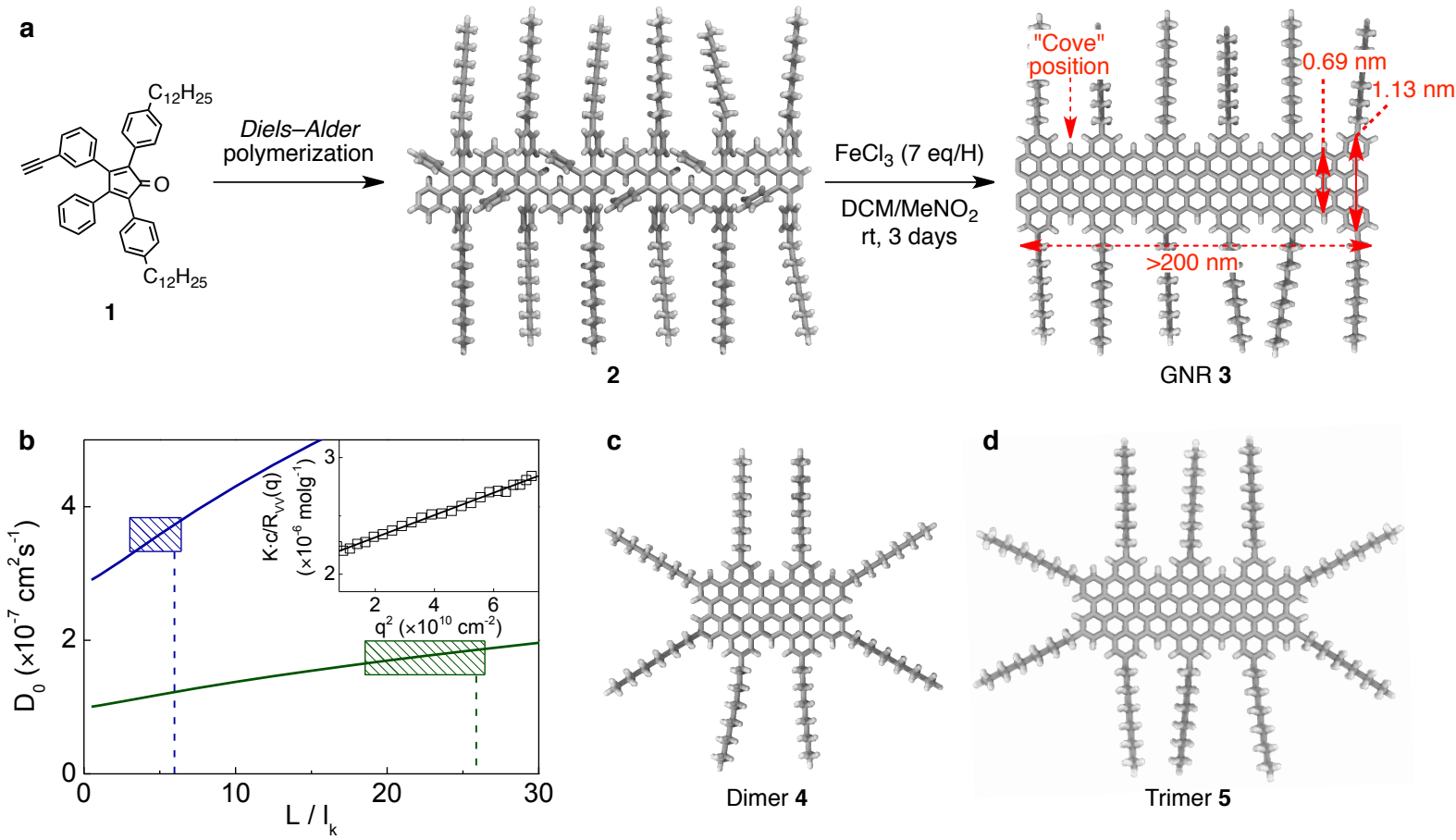

Figure 1. Structures of compounds and light scattering characterization of precursor 2. a, Schematic synthetic route to longitudinally extended GNR 3 via $A B$-type Diels-Alder polymerization of monomer 1. Precursor 2 was "graphitized" into GNR 3 by intramolecular oxidative cyclodehydrogenation. $\mathbf{b}$, Translation diffusion $D_{0}$ of precursor $\mathbf{2}$ as a function of the flexibility ratio $L / l_{k}$ for two different contour lengths $L$ (green line: $480 \mathrm{~nm}$ and blue line: 110 $\mathrm{nm}$ ) and Kuhn segment length, $l_{k}$. The vertical lines correspond to the values of $L / l_{k}$ obtained from eq. 13 in the Supplementary Information; inset: Normalized static light scattering intensity (the size of the symbols captures the experimental error) at different wave vectors represented by eq. 11 in the Supplementary Information yields the molecular weight $M_{\mathrm{w}}$ and the radius of gyration $R_{\mathrm{g}}$ of precursor 2 . In addition, access to the translation diffusion $D_{0}$ (main plot) reveals an unexpectedly large $l_{k} \sim 18 \mathrm{~nm}$ (about 25 repeat units) for precursor 2. c, Structure of dimer 4. d, Structure of trimer 5. Structures of GNR 3, dimer 4, and trimer 5 were optimized by Merck Molecular Force Field 94 (MMFF94) calculations; grey, carbon; white, hydrogen. 
a
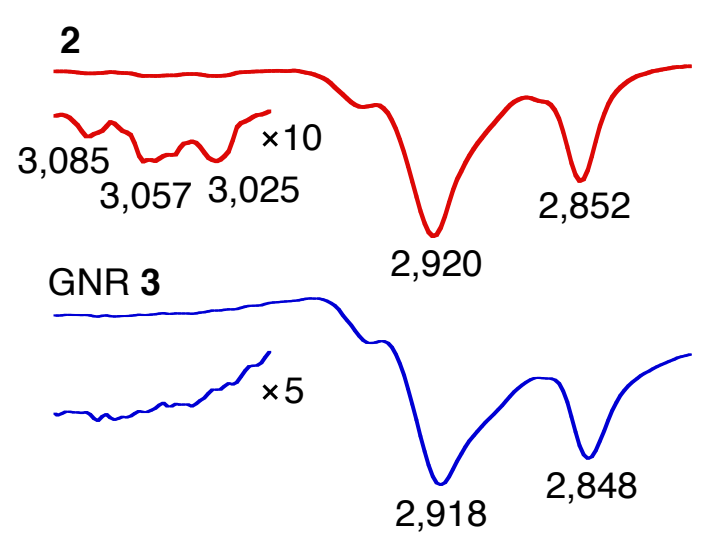

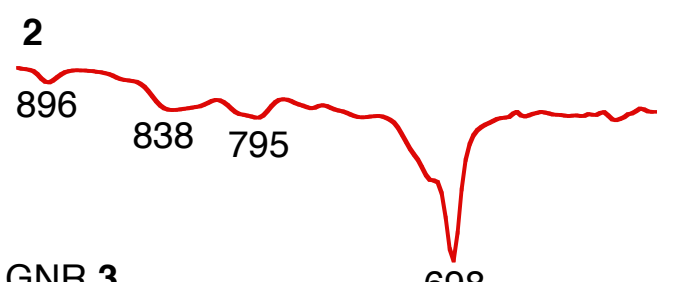

GNR 3

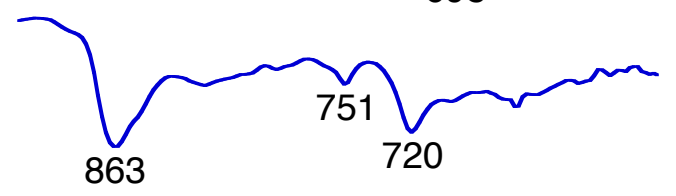

$\begin{array}{cccc}3,100 & \begin{array}{cc}3,000 & 2,900\end{array} & 2,800 \\ & \text { Wavenumber }\left(\mathrm{cm}^{-1}\right)\end{array}$

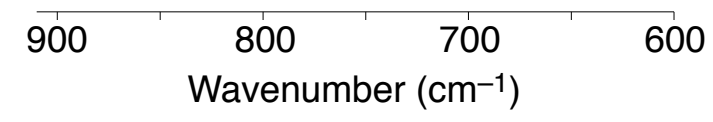
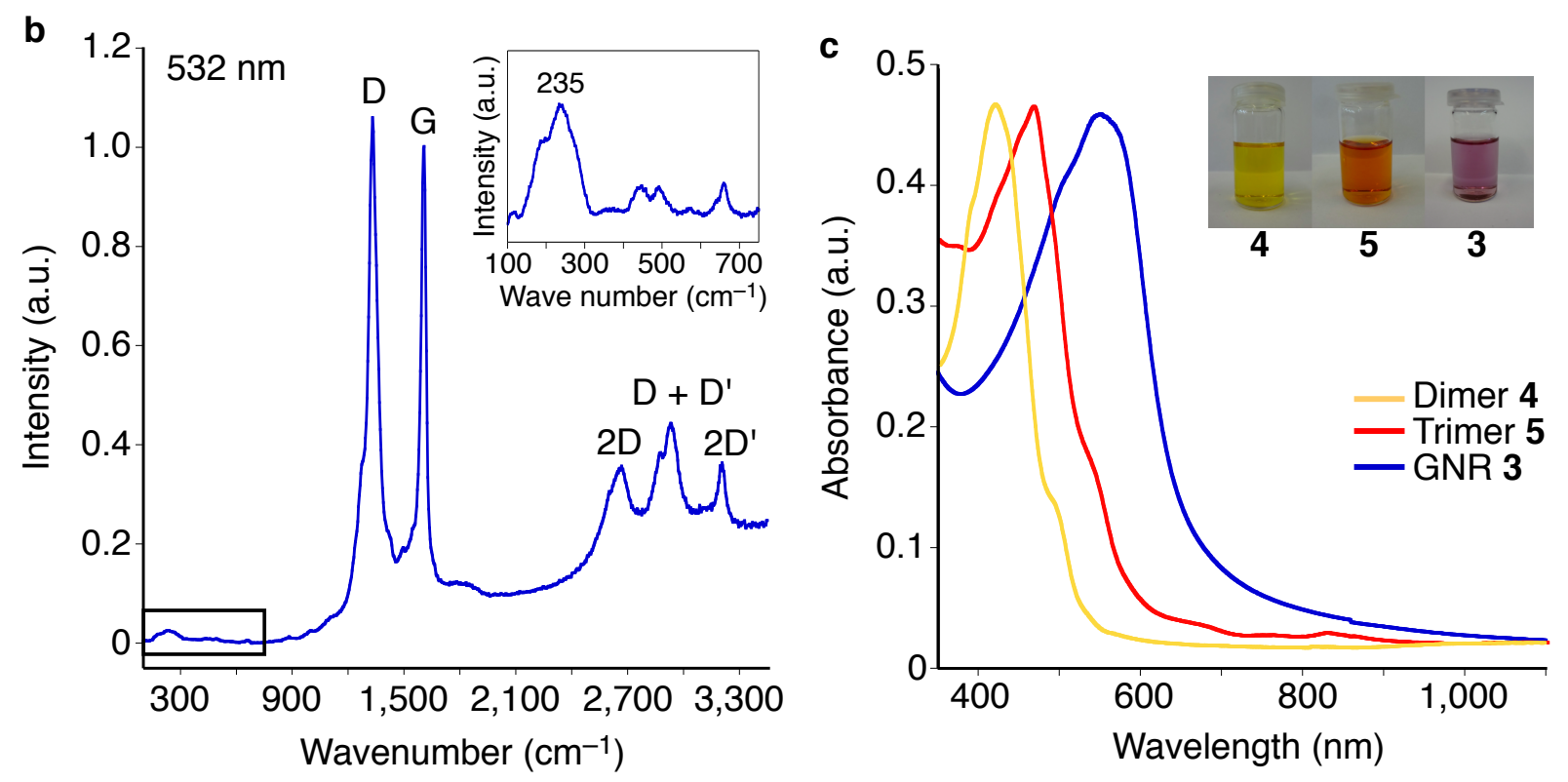

Figure 2. Spectroscopic characterization of GNR 3. a, Representative FTIR spectral regions of polyphenylene precursor 2 (red line) and GNR 3-I (blue line), showing disappearance of the bands derived from mono- and di-substituted benzene rings upon the "graphitization". $\mathbf{b}$, Raman spectrum of GNR 3-I measured at $532 \mathrm{~nm}(2.33 \mathrm{eV})$ on a powder sample with laser power below $0.1 \mathrm{~mW}$. The inset shows a magnified area of the spectrum (black square), displaying a peak from the radial-breathing-like mode (RBLM) at $235 \mathrm{~cm}^{-1}$. Observation of the width-specific RBLM corroborates the high uniformity of the GNRs c, Normalized UV-vis absorption spectra of GNR 3-I (in NMP, blue line) in comparison with those of dimer 4 (in THF, yellow line) and trimer 5 (in THF, red line). The optical bandgaps of dimer 4, trimer 5, and GNR 3 are 1.88, 2.24, and $2.09 \mathrm{eV}$, respectively, based on the absorption edges, demonstrating the lowering of the bandgap upon the longitudinal extension; inset: photographs showing dispersions of $\mathbf{3}, \mathbf{4}$, and $\mathbf{5}$. 

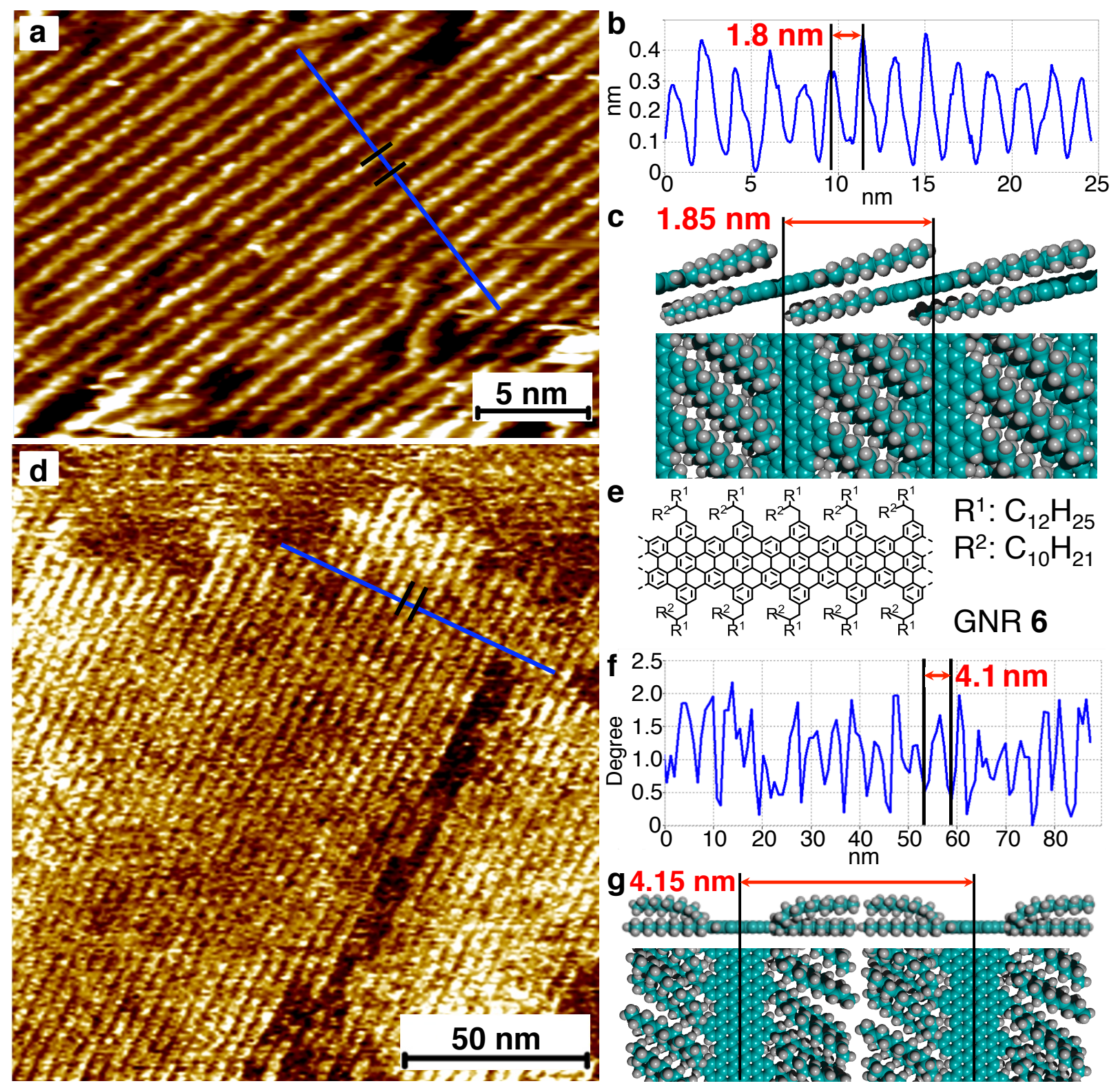

Figure 3. STM and AFM characterization of GNRs 3-I and 6. a, STM image of GNR 3-I on HOPG (dry film), demonstrating a well-organized self-assembled monolayer of straight and uniform nanoribbons of up to ca. $60 \mathrm{~nm}$ in length. b, Line profile (along the blue line in Fig. 3a) showing the periodicity of the structures formed by GNR 3-I on a HOPG surface, which indicates partial stacking of the GNRs. c, Molecular model of partially stacked GNR 3-I; blue, carbon; grey, hydrogen. d, AFM phase image of GNR 6 on HOPG (dry film) demonstrating a highly organized self-assembled monolayer of straight and uniform nanoribbons of over $200 \mathrm{~nm}$ in length. e, Molecular structure of GNR 6. f, Profile (along the blue line in Fig. 3d) of the AFM phase image of GNR 6, displaying the periodicity in agreement with the expected width of the GNRs including the alkyl chains. g, Molecular model of GNR 6; blue, carbon; grey, hydrogen. 

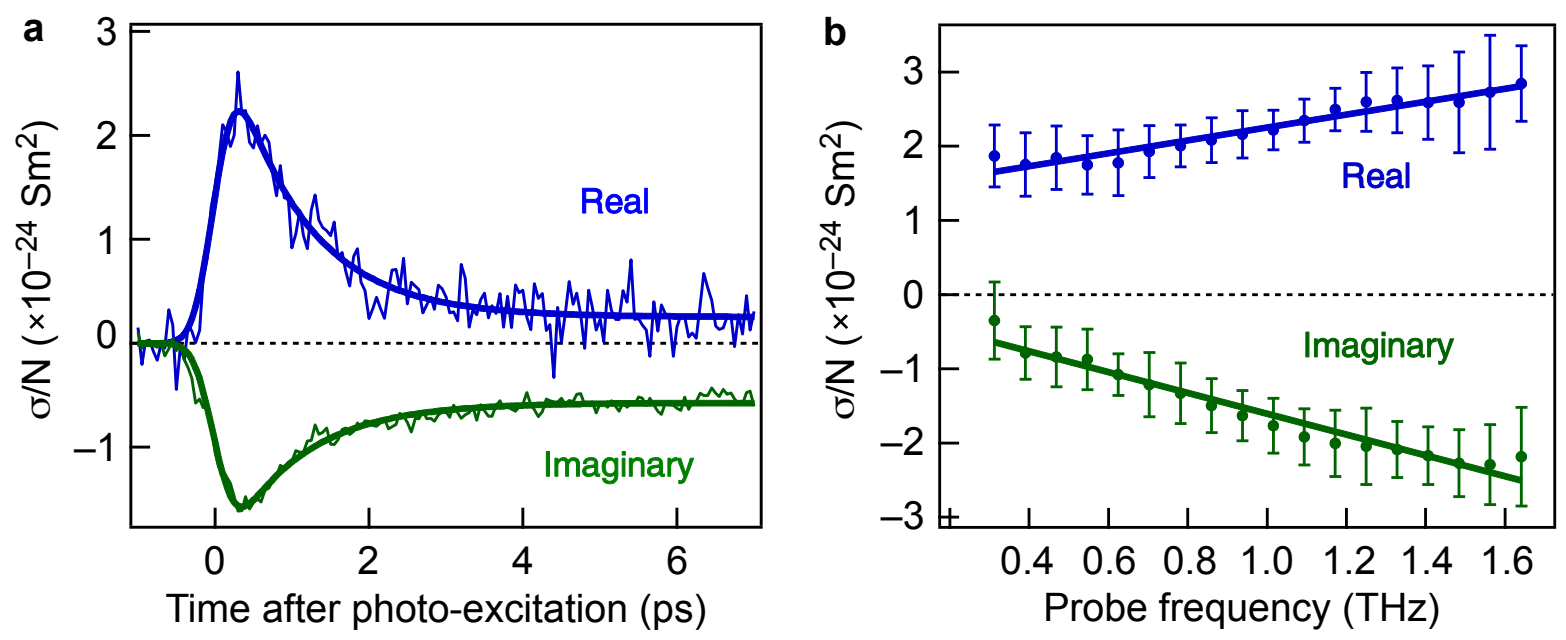

Figure 4. Ultrafast photoconductivity of GNR 3-II. a, Real and imaginary components of the complex photoinduced conductivity of GNRs dispersed in TCB as function of time after excitation. Excitation wavelength was $400 \mathrm{~nm}$. A quick rise in both real and imaginary conductivity is observed after excitation at time 0 , followed by a slower decay. Real conductivity is an indication of photoexcited free charge carriers present just after excitation. Thick solid lines represent simulations revealing free carrier lifetimes of $\sim 1$ ps. b, Frequency resolved complex photoconductivity of the GNR dispersion scaled to initial surface excitation density $N$. The frequency resolved conductivity was measured $300 \mathrm{fs}$ after excitation, at the peak of the photoconductivity, at a fluence of $4.3 \times 10^{18}$ photons $/ \mathrm{m}^{2}$. Solid lines through the data points are guides to the eye. Error bars on the conductivity in $\mathbf{b}$ show plus/minus the standard deviation obtained from 15 consecutive measurements of the $\mathrm{THz}$ waveform. Systematic errors, for instance in the independent determination of $N$, can also affect the magnitude (not the shape) of the scaled conductivity. We estimate the possible magnitude of the systematic errors to be about $25 \%$. Peak magnitudes in $\mathbf{a}$ are scaled to the frequency averaged conductivities of $\mathbf{b}$. 